\title{
Palliative Care in Emergency Medicine: Past, Present, and Future
}

\author{
Moderator: Tammie E. Quest, M.D.1 \\ Participants: Garrett K. Chan, Ph.D., ${ }^{2}$ Arthur Derse, M.D., J.D. FACEP, ${ }^{3}$ Susan Stone, M.D., M.P.H., \\ Knox H. Todd, M.D., M.P.H., ${ }^{5}$ and Robert Zalenski, M.D. ${ }^{6}$
}

Tammie E. Quest, M.D. (Moderator): It is a pleasure to speak with all of you, my esteemed colleagues in emergency medicine, regarding palliative care in the emergency department. You have led this field for over a decade in various capacities and settings, and most of you are also advisory board members for the Improving Palliative Care in Emergency Medicine [IPAL-EM] advisory board, which is sponsored by the Center for Advanced Palliative Care and The Olive Branch Foundation.

I would like to begin this roundtable discussion by asking members of the group to comment on why palliative care is an important area for emergency medicine, and, specifically, what significant signs of progress you have witnessed over the last 5 , or even 10 years? It seems as though our trajectory of growth has been particularly rapid in the last 5 years. Dr. Todd, would you like to start us off?

Knox H. Todd, M.D., M.P.H.: Certainly. Early in my career I was very interested in symptom management and identifying emergency department patients' unmet pain care needs. Competence in palliative care also became very important, because in clinical practice, emergency physicians were frustrated by our lack of ability to meet these unmet pain and symptom management needs, particularly when they included decisions involving end-of-life care.

In 2006, I read a Wall Street Journal article publicizing the work at Virginia Commonwealth University by J. Brian Cassel and others and emphasizing the role of palliative care in controlling medical costs. I contacted Brian and talked with him specifically about his emphasis on the emergency department in palliative care. At that time, the growing palliative care field was interested in the emergency department as a place to identify those with unmet palliative care needs.

Soon after that, at the annual Society of Academic Emergency Medicine meeting in 2006, we held our first workshop to identify where we could contribute emergency medicine research to the field of palliative medicine and how we could train others in emergency care in palliative care core con- cepts. Both J. Brian Cassel from VCU and Steven Pantilat from UCSF contributed to this conference. We have really come a long way since that time.

Dr. Quest, you were one of our early recruits at Emory University and around that time you were developing a specialty for teaching emergency physicians how to deliver bad news, and then began working actively with the Grady Memorial Hospital hospice and subsequently pioneered the palliative care service at Grady Memorial Hospital. A number of others around the country at the same time realized that this was a very important area for emergency medicine to be involved in. I think the growth of emergency medicine palliative care derives from the critical unmet needs that we see our patients experience in our day-to-day practice of emergency medicine.

Robert Zalenski, M.D.: Thank you for this opportunity, and in response to your question, I believe that we must acknowledge that American mainstream medicine really hasn't fully grasped that there are many people who are very sick and feeling bad on a daily basis. Sometimes such people are absolutely so miserable from pain, shortness of breath, or GI disturbances that they really feel like they want to die-not because they are suicidal-but because their state of life is so misery-full that they view death as a desirable alternative. In fact, this is happening all over the country in outpatient clinics right now.

Yesterday I saw a person who had received the benefits of wonderful survival from ovarian cancer and is still alive 9 months after being diagnosed with stage IV cancer. But the problem is that she has not slept for more than 2 consecutive hours in the last 4 months because of agonizing pain, and this is despite what her caregivers are giving her in terms of morphine, and immediate release medications. So she has been poorly managed and is absolutely miserable, and this is cascading to the whole family.

Now picture the fact that such patients often come into the emergency department because they are desperate. And then

\footnotetext{
${ }^{1}$ Department of Veteran's Affairs, Emory Palliative Care Center, Emory University School of Medicine, Atlanta, Georgia.

${ }^{2}$ Emergency Department Clinical Decision Unit, Stanford Hospital and Clinics, Stanford, California.

${ }^{3}$ Center for Bioethics and Medical Humanities, Institute for Health and Society, Medical College of Wisconsin, Milwaukee, Wisconsin.

${ }^{4}$ Palliative Care and House Calls Program, Region 2, HealthCare Partners, Los Angeles, California.

${ }^{5}$ Department of Emergency Medicine, The University of Texas M.D. Anderson Cancer Center, Houston, Texas.

${ }^{6}$ Center to Advance Palliative Care Excellence, Wayne State University, Detroit, Michigan.
} 
what happens? Emergency physicians from the past were not oriented to, "How can I help you with your crisis?" but rather, "Do you have a disease that I can fix and preferably with an injectable drug?"

That viewpoint still permeates, but not to the same extent as in previous times. That was the lay of the land, particularly in the years from 2000 to 2005. Because at the Detroit Receiving Hospital and also at the Detroit VA we have cultivated palliative care in many ways, including our medical school curriculum, and we have a fellowship in palliative care. We have trained over 15 fellows already, and we were just approved 5 years for another 15 . We have been making advances, and now I see that there is a definite change in approach.

In fact, there has been a major change, and doctors are getting onboard with the concept that I am not going to give you the one-size-fits-all treatment in terms of tests and protocols at our hospital, but we are going to actually allow you to give voice to your individuality. We are going to assess you not solely with the idea of fixing your disease, but also of helping you feel better. We are also going to give you a reasonable amount of information so you can make good decisions. In other words, we move from informed consent to informed choice.

This is a very favorable and positive development in the emergency department. But when I explain this in seminars to my emergency attendings, they say that this is nothing short of a revolution because this is not how medicine has been done. What you have in the outlying world is a group of private attendings who think of palliative care as meaning two things: one, you are either trying to kill my patient; or two, you are trying to steal my patient.

We are not trying to do either. We are trying to serve the patient so their days in longevity can be matched by improvements in quality and really help them have a good quality of life. So I am encouraged with what I have seen, but the fact that you can live in the United States in a supposedly great health care system and suffer endlessly without anyone to help you unless you stumble across them or you know somebody in the business is deplorable and must change.

Tammie E. Quest, M.D.: Thank you. Dr. Stone, you have worked in many different environments in palliative care and emergency medicine and outside of emergency medicine in palliative care. Can you share your perspective and the changes you have seen?

Susan Stone, M.D., M.P.H.: Dr. Quest, when you and I started doing this work in palliative care in emergency medicine people thought we were insane. They thought it was heresy, and we did not talk about death in emergency medicine. But we have made a huge shift from that attitude from our colleagues to our colleagues now becoming boarded in palliative care.

Palliative care is not a dirty word among our colleagues now. Sometimes there is confusion about what it means, but overall it has gained enough momentum in emergency medicine that people in emergency medicine are actually interested in becoming certified in hospice and palliative medicine practice.

The IPAL-EM was a huge step forward, as well as the Education in Palliative and End of Life Care-Emergency
Medicine Project [EPEC-EM], which gave us the opportunity to have educational curricula and implementation tools-where we could really show emergency providers how palliative care in the ED can be taught and practiced. In the beginning, emergency physicians thought we were there to put every patient on hospice care. But now we are getting patients much further upstream where we are able to manage a lot of their symptoms, and it is often not a hospice situation.

Right now the ACO [Accountable Care Organization] model is huge. Fee-for-service medicine is going to be dying down after the financial catastrophe it caused. We know we are 40th in health care quality in the world, and we spend most of our GNP on health care. I think the ACO model is going to be good for palliative care because payers are saying, "Listen, we do not want you wasting money on care that is truly futile, and unnecessary, and the patients are suffering."

Patients have a lot more to say now about quality than ever before. The other big step I would say is that we actually have quality data and quality networks developing to help us define quality standards for palliative care. These things all trickle down into the emergency department. The field is a whole world from where it was. Do I think we have a lot more progress to make? Absolutely.

Everybody is starting to see that the palliative care approach improves quality even in the emergency department. The EPEC-EM Project helped us teach people how to provide emergency palliative care, which is quite different than the palliative care on the ward or in outpatient settings.

Tammie E. Quest, M.D.: Thanks Dr. Stone. Dr. Derse, you have been practicing emergency medicine for 3-plus decades now. Please give us a short overview of where this field was 5 or 10 years ago and where it is now from where you sit as a non-palliative care specialist and as a bedside emergency clinician working side-by-side with providers every day trying to do this work.

Arthur Derse, M.D., J.D. FACEP: There is an understanding of palliative care in the emergency department now. Medical students and residents from medical schools where palliative care has been taught come with an understanding that palliative care is a service that can help and is available. I think the fact that hospice and palliative medicine was recognized as a subspecialty of emergency medicine has really acted as a clarion call to the rest of emergency physicians.

An analogy is that not every emergency physician has to be a toxicologist, but you need to know the principles of toxicology to practice good emergency medicine. Similarly, you need to know the principles of hospice and palliative medicine to be able to practice emergency medicine well. This is now being recognized as part of the competency of an emergency physician.

I do think that palliative care is being integrated in the emergency department in a way that it had not been 5 or 10 years ago. That having been said, I do think that it is still in the first phase of that integration. Palliative care has still not been well understood and accepted by the majority of ED physicians. This is also true for many oncologists, cardiologists, and nephrologists who are not including palliative care as part of their dayto-day discussions with patients. So there is still a lot of work to do among many specialties, including ours. 
Tammie E. Quest, M.D.: Thanks. We are going to move on to the topic of gaps and challenges. A recent study published by Dr. S. Lamba and colleagues shows that emergency department-initiated consults were most often for imminently dying patients and oftentimes involved patients that were young with unexpected and serious illness. ${ }^{1}$

We all know that palliative care consultation can be useful at many points in an illness other than care of the imminently dying patient. Do you see this changing in emergency medicine and are there some special challenges? Dr. Chan, what is your response to this question?

Garrett K. Chan, Ph.D.: There are special challenges in implementing palliative care. The focus of emergency medicine and nursing has really been to evaluate and stabilize patients and has been on resuscitation and diagnosis and treatment of various conditions.

Palliative care helps us see the patient as a whole person, understanding what their preferences or choices are for their goals of care. That switch in philosophy is going to be quite challenging as we move forward. However, I believe that there is, as Dr. Derse was saying, a movement toward educating professionals, both in nursing and in emergency medicine, to evaluate this concept of humanistic care that we are moving toward.

The other thing about palliative care that resonates with emergency nurses and physicians is the concept of utilizing an interdisciplinary team. Throughout the whole emergency department stay, patients and families are cared for by physicians and nurses concurrently, as well as by social workers, chaplains, and other health care providers such as physical therapists. So looking at how we save our health care resources and meet the patients' needs with the available resources and appropriate resources is the next area that we will embark on, especially with the advent of ACOs and accountable payments that are value-based versus just fee-forservice.

Tammie E. Quest, M.D.: Thank you. One of the things, Dr. Derse, that we have talked about in regards to emergency medicine and that is somewhat unique to our specialty is the topic of ethical and legal challenges that emergency clinicians find that interface in the emergency department and that may not be felt by other clinicians who are less concerned with medico-legal risk. Where have we been with this over the last decade, and are we in a better place now than we were before? Do you think medico-legal concerns still hinder emergency clinicians from discussing end-oflife care that may include discussions to forego lifesustaining interventions and use of medications for symptom control in the ED and upon discharge?

Arthur Derse, M.D., J.D. FACEP: I do think that there has been great progress made. Emergency physicians have made an important sea change. Years ago we would tend to err on the side of intervention, assuming that everybody needed resuscitation, everybody needed aggressive treatment, and not be bothered gathering any information to the contrary, and admit the patient to the hospital and have it all sorted out in the ICU. I think emergency physicians now understand that treatment limitation at the end of life-as part of an advance care plan-is an appropriate option. Not as many ED physi- cians are comfortable with stopping treatment in the emergency department, but they understand that advance care planning, pre-hospital DNRs, and POLST documents-that is, physician orders on life-sustaining treatment-that are valid in many states, all have utility for emergency physicians in making treatment decisions about patients. They understand that it is no longer true that you should always err on the side of continued aggressive treatment. So that has changed.

But it is also true that we tend to think about our interventions as being authorized through the exception to informed consent-the emergency exception, where there is as a life- or limb-threatening emergency; the patient is not decisional; there is no legal surrogate who can speak; time is of the essence and a reasonable person would consent. When all of the above apply, then we are authorized to intervene without consent, but for the majority of ED cases, not all of these essential elements are present. And when one or more elements are missing, we do not have authorization to intervene without consent. For instance, there may be an advance care plan that we should know about. There may be someone who can speak for the patient. Even if we do intervene, we need to inform not just about the benefits of treatment, but also about the burdens, the risks of the particular treatment, as well as the fact that if the treatment is no longer achieving the goals that the patient and the physician have set or if the patient no longer wants the treatment because it is burdensome or they just are tired of it, the treatment will be stopped.

That important information needs to be conveyed at the beginning of any intervention that we plan to implement, so that when the time comes for our colleagues in palliative medicine to talk about stopping those treatments on those bases, family members may be reminded of those original conversations and understand that the time to stop has been reached.

Then the other area that is a huge challenge is the issue of futility. There are reasons why emergency physicians should provide good life-sustaining treatment when indicated but not necessarily provide all life-sustaining medical treatments for all patients. Many emergency physicians are reluctant to withhold requested life-sustaining treatment-despite their considered medical judgment that the intervention won't work-because they fear legal consequences, even though there are very supportive statements and policies by both the American College of Emergency Physicians and the American Medical Association supporting their medical judgment. In addition, hospitals may have their own applicable supportive policies. Nevertheless, emergency physicians have the professional responsibility to make decisions about appropriate emergency interventions.

The other thing that I think is changing is the use of opioids, benzodiazepine, and other medication use in end-oflife care in the emergency department. Emergency medicine physicians need to be reminded that just as we understand the importance of their use for symptomatic relief in the ED they are just as important and useful in end-of-life ED patients.

There is a growing consensus among the Federation of State Medical Boards and the DEA in recognizing that pain needs treatment. These regulatory agencies are also worried about a growing epidemic of individuals who 
have narcotic addiction problems. But ED physicians should recognize that this concern about addiction should not pertain to those patients who are facing the end-oflife in pain.

All of these are areas where there has been a significant change in practice. That having been said, there are some areas where we still have challenges left. Emergency clinicians will need to become part of the team that, when indicated, discusses the option of palliative care. There have been states that have passed "right-to-know" laws that require the physician to inform the patient of the option of palliative care. In fact, in California it is only required if you are asked by the patient, but in New York it is required, even if the patient does not ask.

Informing the patient of the option of palliative care may sometimes fall to emergency physicians because studies shows that for our colleagues in oncology, nephrology, and cardiology, palliative care is often not presented as an option. It may be the emergency physician who will be ethically and legally responsible to present the option.

A final legal issue in end-of-life care is the issue of physician-assisted suicide. It only applies in three states currently-Oregon, Washington, and Montana. But it may continue to grow as a phenomenon. Massachusetts will soon have a referendum whether to legalize this practice as well. The emergency physician's role in this practice may be limited, but there may be patients who present to the emergency department who have availed themselves of this choice. Emergency physicians will need to know how to respond and, if the patients have acted in accord with the law, will need to provide palliative support rather than acute intervention. That is something that emergency physicians will have to work out in the handful of states that have legalized this option.

Tammie E. Quest, M.D.: The emergency department team consists of many different providers and the nurse/physician bedside team is really central to patient care. Dr. Chan, please comment on the role of the emergency nurse in palliative care and how that can be an important aspect of the team.

Garrett K. Chan, Ph.D.: As I mentioned before, the nurse/ physician dyad is an important construct within the emergency department. Unlike other parts of health care, we have close working relationships that really are interdependent. Nurses help physicians inform the differential diagnosis and the problem areas that are identified through their assessments at triage and even at the bedside. In addition, they get to spend more time with patients and families in those states that have nurse/patient ratios whereby more information can be gathered about goals of care and that can be shared with the team.

The nurses also play a tremendously important role in figuring out possibilities for care in the context of the patient's illness trajectory and the resources that are available to meet those needs. Nurses are the eyes, ears, and hands oftentimes of physicians and through their assessment and reassessment after an intervention has been provided, specifically in symptom management, they help the whole team understand the physiologic or symptom management needs, so plans of care can be adjusted accordingly.
When we look at other members of the health care team such as case managers in the emergency department, these people can help with resources for discharge or even admission and match appropriate levels of care with the patient's needs. This is also an important concept that is beginning right now, especially with health care reform.

The chaplain and the social worker delve more into the psychosocial needs of not only the patient but also of the family and looking at the family as part of the patient's unit of care. It is really in the domain of palliative care, and we are making huge strides as we introduce palliative care to the emergency department.

Tammie E. Quest, M.D.: This past year the IPAL-EM Project has worked to create tools and technical assistance monographs to encourage the effective integration of palliative care in the emergency department with or without the presence of a palliative care consultation service. Key domains that have been identified have been adapted from the National Palliative Care Consensus Guidelines with companion needs assessment in eight key domains that pertain to the emergency department. Some of those include screening patients for palliative care needs in the emergency department, documentation honoring advance care plans, multidisciplinary care plans for patients in pain, grief and bereavement plans for patients that die in the emergency department, and recognition that caregivers need support, as well as spiritual support for the family unit. And then, importantly, care of the imminently dying patient.

I would like for the group to comment very specifically on any examples that they have been able to see in terms of implementation in these domains of actual projects that have been carried out or that have worked in your care setting.

Susan Stone, M.D., M.P.H.: I have been impressed by the Coalition for Compassionate Care, which has really taken charge in California of POLST education to our pre-hospital providers into the ED, and this falls on the turf of the emergency department.

We are starting to identify POLST forms and actually read and listen to them because I think we are all pretty aware that many patients come in without a directive at all. There is community-wide education with providers in the community pre-hospital on the POLST. I think this has been a really important step.

Robert Zalenski, M.D.: I have the privilege of being the medical director of palliative care for the Vanguard Health System, which is a system of at least 28 hospitals in five markets, including two of the largest cities in the United States.

We are going to lay down a palliative care directive, which will require a staff member of the ED and a staff member of the ICU to be versed in palliative care. We will train a cadre of people who will be able to do an initial assessment looking at patients with uncontrolled symptoms, patients with poor function, and to look immediately for patients with evidence of advance care planning if they are not decisional. If they are decisional, then we will talk to them or to their decisional surrogates to get a specific understanding 
of their goals and plans of care and their wishes. We want to integrate this into a routine assessment in the emergency department.

We are actually going to charge the emergency department to come up with this in the same way that they have been very good at routinely assessing if the patient comes in as a trauma code. That is our system intervention we have on the table now.

Knox H. Todd, M.D., M.P.H.: I would just comment that although we have been speaking of some fairly large advances in our consensus about emergency medicine palliative care standards and guidelines, there is a huge variation between emergency departments palliative care practices. In a sense, all palliative care is local. If one can identify a specific individual champion within an emergency department, whether it is a physician, nurse, social worker, chaplain, or someone in administration, and identify specific stories that have meaning to the emergency department staff-that is how you really get things started.

The beauty of the IPAL-EM resource is that it can provide very targeted education. An emergency provider can go to the site and find specifically what is pertinent to his or her practice setting and utilize that to make strategic, focused advances.

I recall one case at a hospital in New York that involved a bereaved family not treated with respect, and forced to grieve in an undignified atmosphere. This really drove an intervention that made a great deal of difference in a single site. Although we have talked about large-scale standards interventions, this is a one-emergency-department-at-a-time project.

Tammie E. Quest, M.D.: That is a great transition to my next topic, which is that once we have those impactful programs and processes in place we need to think about how we are going to measure the impact of that work. Emergency departments provide an important public service by providing emergency care 24 hours a day without discrimination by social or economic status. One of the key expectations of the ED is the ability to provide immediate access with stabilization for those patients who have an emergency medical condition.

We have heard lots over the last decade around overcrowding. Palliative care in the ED is often thought of as time-consuming and not the right time or right place. The IPAL-EM group has worked to develop and make the case, as well as an ED evaluation of palliative care through metrics and quality measures, to address thinking about that value argument. When we think about real, tangible processes and metrics that are looked at in the emergency department, can anyone comment on the value argument for providing good palliative care in the emergency department when it really comes down to some of the things that we measure in the ED?

Knox H. Todd, M.D., M.P.H.: To the extent we measure symptom burden in a standardized fashion, and I would use pain as an example, improvements in our practice should improve the outcomes for those metrics. The same can be said for a broad variety of symptoms if measured through standard symptom distress instruments.
Another measure that we will increasingly be held accountable for is the phenomenon of emergency department or hospital readmissions and revisits. Palliative care can provide a higher quality of life for a patient, while at the same time, prevent what is often an endless cycle of patients who move from either the home or nursing home setting to the emergency department and back again without a good deal of thought being given to the more important decisions that need to be made about treatment in light of the patient's and family's goals of care.

Robert Zalenski, M.D.: We did a single-hospital study we presented where we examined patients who came from nursing homes and were evaluated in the ED and then admitted anywhere in the hospital. Almost every patient-I think it was $99 \%$ - had palliative care needs and they actually met the standard hospice criteria for their major diagnosis. But we found though that only about $20 \%$ of those, had goals-of-care discussions. For those that did, there were changes and plan disposition and critical care choices that were made.

We serve, as we always have, as a gateway to getting off on the right foot and matching the kind of care, testing, and treatment they will receive with their goals. If we improve on that sum every year and every quarter by measuring the outcomes related to that, we will not only provide outstanding service and quality, but we will also contribute in a substantial way to the health care equation, which is the ratio of value to cost. What many people want is care that is tendered helpfully and not necessarily the most costly procedure that money can buy.

Garrett K. Chan, Ph.D.: Going back to the example about one hospital in New York where a family was forced to grieve in an undignified manner. One of the key skills of people who are trained in palliative care, and also people who have taken palliative care education, is the ability to communicate.

As hospitals are forced to look at patient satisfaction scores as an indicator for reimbursement in the setting of valuebased purchasing, the better equipped emergency clinicians are to understand all aspects of palliative care the more we might see an affect on patient satisfaction and the quality of care that is delivered.

Tammie E. Quest, M.D.: Thanks Dr. Chan. Moving on to our final topic, in the next 10 years, what would the group like to see with respect to implementation of palliative care in the emergency department and what would success look like?

Susan Stone, M.D., M.P.H.: In the next 10 years I would like to see all of California hospitals with a palliative care service. Right now we are over $60 \%$ and $100 \%$ of our county hospitals have palliative care as of this month. So that is good. But success to me would be all hospitals having a palliative care service.

I would also like to see early consults from the emergency department. We know that if we wait until the patient goes to the floor, a consult happens much later. I would like to see more consults coming from the ED earlier on.

We also have to recognize and encourage the non-palliative care physicians and staff to get trained as well because, as you 
know, we are short staffed on palliative care providers. And I would certainly like to see pink POLST forms flowing over the walls of the ED so that everybody's preferences are known and we do not resuscitate a person against their wishes, which still happens pretty commonly because you cannot find an advance directive. But I would like to see the POLST in every home.

Finally, I would also like to see the younger patients who are part of the surfing, skateboarding, and biking culture to have completed something like Five Wishes ${ }^{\circledR}$.

Garrett K. Chan, Ph.D.: Over the next 10 years, I would like to see an improvement in primary palliative care at each individual emergency physician and nurse level. One of the things that drive emergency nurses and physicians is using algorithms to guide care for patients.

Instead of trying to change a whole paradigm of how we approach patients, especially in emergent situations where we do not have a lot of information about the patient or maybe the information is erroneous in a very time-sensitive, pressured situation, if we can change, perhaps, the algorithm of shortness of breath presentation, for instance, should intubation be the next step in severe respiratory distress or should palliative care principles, such as goals of care, be assessed simultaneously. Maybe if we just asked the question, "Is this right for the patient, physiologically or for their disease process, or is this right for the patient because they do not want it?"

If we could just stop and have that 5-second question in our head before we go down a particular path, then that would be a measure of success. We need to focus on the whole patient in terms of goals of care, family caregiver burden, and look at other pause points within the algorithm of the care delivery. That would be a measurement of success at the primary palliative care level.

Arthur Derse, M.D., J.D. FACEP: A measure of success would be one, emergency physicians actually discussing palliative care with appropriate patients, even if the topic has not come up before, and two, having a documentation of the risks, burdens, and benefits of any proposed life-sustaining interventions.

Another quality measure would be having more access to palliative care by emergency medicine because one of the things that I have heard from some emergency physicians is that it is really hard to get a palliative care consult in the emergency department. It is difficult to have palliative care coverage able to provide a consult quickly in the ED 24/7 in some centers. But if emergency physicians were able to access the palliative care service when they need it, they would use the consultation service more.

Finally, one of the best quality measures would be, even if you cannot get palliative care support to help at the ED bedside, that the emergency physician would feel comfortable stopping life-sustaining medical treatment and allowing the patient to have an ethical and comfortable death in the ED.
That is something that is still a major barrier for emergency physicians who are not trained in palliative care concepts.

Knox H. Todd, M.D., M.P.H.: In 10 years, I would like to see a 10 -fold increase in the number of emergency physicians who have received specialized training in palliative care. I would like to see an emergency physician who is well trained in palliative care at each academic training site. I would like to see emergency physicians routinely having a place on the palliative care structure within the hospital and quality metrics for emergency department palliative care incorporated by The Joint Commission.

Robert Zalenski, M.D.: My goals for the emergency department would be to really understand what our role is in the system of care and how we can best participate in the newer goals of having this non-bricks-and-mortar way to keep people healthy. Even more than when people can no longer maintain their function or get back to baseline, that we play an active and vital role in ensuring that they get the kind of transition, welcome, symptom relief, and communications that palliative care is really known for.

What I would love to see is for us to get together like we did in Atlanta one time for the future of emergency medicine to revision what kind of care and emergency medicine would be in the system. I believe there are really two parts. The one part would be toward what we have always done so well, which is saving function, but the second would be toward helping patients who are having acute, traumatic injuries that bring them to the end of their life precipitously and the ability to provide end-of-life care that they deserve. This is going to be a process, and 10 years is probably the right time for the transition.

Tammie E. Quest, M.D.: As the moderator of this session, it means so much for me to have seen over the last 10 years where we have gone and where we have grown and to hear all of you echo that. Thank you so much for your time and insights.

\section{References}

1. Lamba S, Nagurka R, Walther S, Murphy P: Emergencydepartment-initiated palliative care consults: A descriptive analysis. J Palliat Med 2012;15:633-636.

2. Five Wishes ${ }^{\circledR}$. Aging with Dignity. www.agingwithdignity .org/forms/5wishes.pdf (Last accessed July 9, 2012).

Address correspondence to: Tammie E. Quest, M.D. Emory Palliative Care Center Emory University School of Medicine 1462 Clifton Road, Suite 302 Atlanta, GA 30322

E-mail:TQUEST@emory.edu 